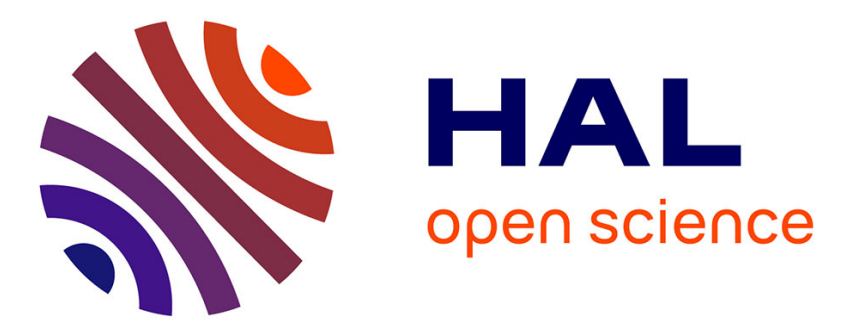

\title{
Dispositif transistorisé pour l'amorçage par étincelle glissante des décharges utilisées comme source de lumière pour la spectroscopie dans l'ultraviolet lointain
}

\author{
H. Turquet de Beauregard, M. Morlais, S. Robin
}

\section{- To cite this version:}

H. Turquet de Beauregard, M. Morlais, S. Robin. Dispositif transistorisé pour l'amorçage par étincelle glissante des décharges utilisées comme source de lumière pour la spectroscopie dans l'ultraviolet lointain. Revue de Physique Appliquée, 1968, 3 (1), pp.25-26. 10.1051/rphysap:019680030102500 . jpa-00242816

\section{HAL Id: jpa-00242816 https://hal.science/jpa-00242816}

Submitted on 1 Jan 1968

HAL is a multi-disciplinary open access archive for the deposit and dissemination of scientific research documents, whether they are published or not. The documents may come from teaching and research institutions in France or abroad, or from public or private research centers.
L'archive ouverte pluridisciplinaire HAL, est destinée au dépôt et à la diffusion de documents scientifiques de niveau recherche, publiés ou non, émanant des établissements d'enseignement et de recherche français ou étrangers, des laboratoires publics ou privés. 


\title{
DISPOSITIF TRANSISTORISÉ POUR L'AMORÇAGE PAR ÉTINGELLE GLISSANTE DES DÉGHARGES UTILISÉES COMME SOURGE DE LUMIÈRE POUR LA SPEGTROSGOPIE DANS L'ULTRAVIOLET LOINTAIN
}

\author{
Par H. TURQUET de BEAUREGARD, M. MORLAIS et S. ROBIN, \\ Laboratoire de Spectroscopie, Faculté des Sciences, \\ avenue du Général-Leclerc, 35-Rennes.
}

(Reçu le 22 septembre 196\%.)

\begin{abstract}
Résumé. - On étudie l'amorçage des décharges de condensateurs dans un tube capillaire où règne un vide poussé, qui sont utilisées comme source de lumière pour la spectroscopie dans l'U.V. lointain. Pour que l'amorçage soit convenable, il faut que l'intensité du courant et l'énergie dépensée dans l'étincelle glissante soient suffisantes. On décrit un dispositif simple entièrement transistorisé permettant d'obtenir un bon amorçage avec une fréquence réglable.

Abstract. - The triggering of discharges of capacitors in a capillary tube with a high vacuum, used as a source of light for the spectroscopy in the far U.V. is studied. For good triggering, the intensity and the energy spent in the sliding spark must be sufficient. A simple device totally transistorised allowing good triggering with an ajustable frequency is described.
\end{abstract}

L'étincelle glissante a été utilisée pour la spectroscopie dans l'ultraviolet lointain comme source d'abord [1], comme auxiliaire ensuite. Elle sert alors à amorcer des décharges dans le vide, soit entre électrodes métalliques rapprochées [2], soit dans un capillaire susceptible de produire une décharge de type Lyman sans introduction de gaz [3]. L'énergie libérée dans l'étincelle glissante est emmagasinée dans un condensateur $\mathrm{C}_{\mathbf{1}}$. Celui-ci peut être chargé par l'alimentation électrique même de la source et déchargé dans l'étincelle par un contact mécanique. Un tel système est généralement bruyant et d'utilisation peu pratique. Il est préférable d'utiliser une alimentation auxiliaire pour la charge de $\mathrm{C}_{\mathbf{1}}$ et un thyratron amorcé par un oscillateur pour provoquer la décharge de ce condensateur. Jusqu'ici, nous utilisions à cet effet un thyratron à gaz (Sylvania $4 \mathrm{C} 35$ ) pour décharger une capacité de $0,07 \mu \mathrm{F}$ chargée à 4 kilovolts [3]. Dans le but de diminuer les dimensions de ce dispositif d'alimentation et de pilotage, ainsi que la tension de charge de $\mathrm{C}_{1}$, nous avons cherché à réaliser un ensemble transistorisé piloté par un thyratron à semi-conducteur.

Le dispositif est représenté figure 1. Le condensateur $\mathrm{C}_{1}$ chargé sous 400 volts est déchargé dans le primaire $\mathrm{du}$ transformateur $\mathrm{T}_{2}$ par l'intermédiaire d'un thyratron au silicium ( $2 \mathrm{~N} 687$ Sesco); celui-ci peut être amorcé très simplement par un transistor unijonction ( $2 \mathrm{~N} 1671 \mathrm{~B}$ Sesco). Ce thyratron à semi-conducteur présente l'avantage de n'avoir qu'une très faible résistance interne pendant la décharge. La fréquence de répétition dépend de la constante de

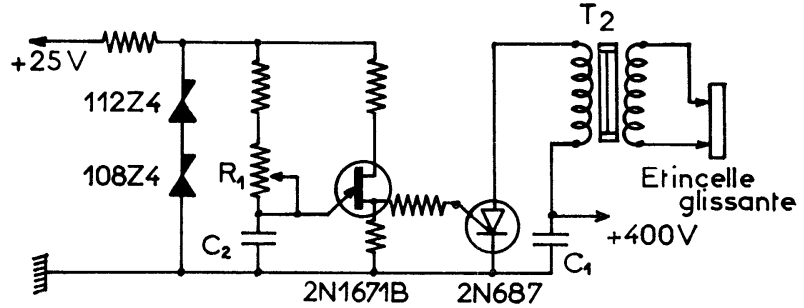

FIG. 1.

temps des éléments qui commandent l'émetteur du transistor unijonction. Cette fréquence est ajustable sur un large intervalle par un choix convenable de la capacité $\mathrm{C}_{2}$ et le réglage de la résistance variable $R_{1}$. Les diodes Zener $112 \mathrm{Z} 4$ et $108 \mathrm{Z} 4$ assurent une stabilisation de la tension d'alimentation de cet oscillateur.

La grosse difficulté réside dans le choix du transformateur $\mathrm{T}_{2}$. Nous avons pensé d'abord à utiliser une bobine d'induction (type bobine d'allumage pour moteur à explosion) ou une bobine T.H.T. de télévision. Ces dispositifs permettent d'obtenir aux bornes de leur secondaire des tensions de plusieurs dizaines de milliers de volts; ils se sont avérés inefficaces pour l'amorçage de nos décharges à des pressions inférieures à $10^{-2}$ torr dans la source. D'autre part, ces hautes tensions présentent l'inconvénient de nécessiter des isolements très soignés, et de ne pas pouvoir être utilisées avec certains types d'étincelles glissantes : par exemple, des lames minces de mica [4] sont rapidement mises hors d'usage. 
Nous avons alors pensé que la haute tension n'était pas la qualité principale pour un bon amorçage et nous avons étudié systématiquement les éléments (noyau et bobinage de $\mathrm{T}_{2}$ ). Une tension de 1500 à 2500 volts à l'instant de l'amorçage apparaît suffisante, mais il est nécessaire que l'intensité du courant dans l'étincelle glissante et la durée $\tau$ de la décharge soient assez grandes (il faut probablement libérer un nombre d'ions suffisant avec une densité suffisante pour que la décharge principale s'amorce correctement). Une intensité maximum de 100 à 200 ampères (limitée par le thyratron $2 \mathrm{~N} \mathrm{687)} \mathrm{nous} \mathrm{a} \mathrm{donné} \mathrm{de} \mathrm{bons} \mathrm{résultats}$ avec une durée $\tau$ de 50 à 200 microsecondes. Avec cette même intensité et des valeurs inférieures de $\tau$, la proportion des décharges principales non amorcées croît très vite lorsque $\tau$ diminue et la source devient instable. Par contre, avec d'autres alimentations plus puissantes utilisant un thyratron Sylvania 4 C 35 [3], nous avons pu obtenir un bon amorçage avec des durées $\tau$ beaucoup plus courtes mais avec des intensités beaucoup plus grandes ( $\tau$ voisin de $5 \mu$ s avec une intensité maximum de 3000 ampères).

Pratiquement, les variables possibles sont la capacité $\mathrm{C}_{1}$ et les nombres de spires $n_{1}$ au primaire et $n_{2}$ au secondaire du transformateur. Le rapport $\frac{n_{2}}{n_{1}}$ est conditionné par la tension d'amorçage lorsqu'on s'est fixé la tension de charge $V_{1}$ du condensateur. Pour $C_{1}$ donné, $n_{1}$ doit être suffisant pour que l'intensité ne dépasse pas la valeur maximum supportable par le thyratron. On pourrait diminuer $C_{1}$ et $n_{1}$, mais si la durée de la décharge diminue trop, l'amorçage devient moins satisfaisant : il faut dissiper une énergie suffisante dans l'étincelle; d'autre part, il n'apparaît pas nécessaire d'augmenter $C_{1}$ et $n_{1}$ au-delà des valeurs nécessaires si l'on veut avoir une alimentation de puissance aussi réduite que possible tout en permettant un fonctionnement à rythme rapide. Nous avons obtenu de bons résultats avec un circuit coupé en tôles d'acier au silicium à grains orientés (par exemple « Silicore » AD 32 Isolectra), dont la section est d'environ $7 \mathrm{~cm}^{2} ; \quad V_{1}=400$ volts, $C_{1}=20 \mu \mathrm{F}, n_{1}=30$, $n_{2}=150$. Les enroulements doivent être réalisés en fil de section suffisante (cuivre émaillé de diamètre $2 \mathrm{~mm}$ par exemple) pour que la résistance du circuit primaire reste faible $(0,05 \mathrm{ohm}$ environ) : l'intensité au secondaire décrôit très vite lorsque cette résistance croît. Il apparaît préférable de bobiner les deux enroulements l'un sur l'autre avec un isolement convenable. Avec les conditions précédentes, la durée de la décharge est de l'ordre de $100 \mu$ s avec une intensité maximum de 100 ampères. Dans ces conditions, l'amorçage des décharges dans un tube capillaire [3] est très bon pour une pression de $10^{-4}$ torr avec une fréquence de 15 hertz; à rythme plus lent, exigeant une vitesse de pompage moins rapide dans la source, on peut amorcer facilement à des pressions de $10^{-5}$ torr. Des essais de stabilité à fréquence élevée $(15$ à $20 \mathrm{~Hz}$ ) obtenus en arrêtant le défilement des longueurs d'onde du monochromateur sur le sommet d'une raie ont montré que celle-ci était au moins aussi bonne qu'avec les anciens dispositifs d'alimentation [3].

\section{BIBLIOGRAPHIE}

[1] Romand (J.), BALlofFe'T (Mlle G.) et Vodar (B.), C.R. Acad. Sc. Pavis, 1955, 240, 412.

[2] Romand (J.), BALLOFFET (Mlle G.) et KIEFFER (B.), Spectrochim. Acta, 1962, 18, 791.
[3] Morlais (M.) et Robin (S.), Applied Optics, 1967, 6, 109.

[4] Damany (H.), Roncin (J. Y.) et Damany Astoin (N.), Applied Optics, 1966, 5, 297. 